\title{
Liraglutide improves hepatic insulin resistance via the canonical Wnt signaling pathway
}

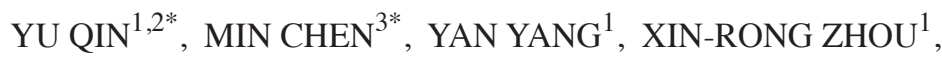 \\ SHI-YING SHAO ${ }^{1}$, DAO-WEN WANG ${ }^{1}$ and GANG YUAN ${ }^{1}$
}

\begin{abstract}
${ }^{1}$ Department of Internal Medicine, Tongji Hospital, Huazhong University of Science and Technology, Wuhan, Hubei 430030; ${ }^{2}$ Department of Endocrinology, Affiliated Hospital of Jiangsu University, Zhenjiang, Jiangsu 212000; ${ }^{3}$ Department of Geriatrics, The First Hospital of Jiangxia, Wuhan, Hubei 430030, P.R. China
\end{abstract}

Received March 7, 2017; Accepted November 23, 2017

DOI: $10.3892 / \mathrm{mmr} .2018 .8737$

\begin{abstract}
Liraglutide, a modified form of glucagon-like peptide-1 (GLP-1), is used in the treatment of diabetes mellitus. However, the underlying mechanism by which liraglutide improves liver insulin resistance remains to be elucidated. The proto-oncogene Wnt (Wnt) signaling pathway has been reported to be associated with glucose and lipid metabolism. Using in vivo and in vitro models of diabetes and insulin resistance, it was investigated whether the beneficial effects of liraglutide on liver glucose metabolism are mediated by the Wnt signaling pathway. The results of the present study demonstrate that body weight, fasting blood glucose, insulin levels and the homeostasis model assessment for insulin resistance were markedly decreased in $\mathrm{db} / \mathrm{db}$ mice treated with liraglutide compared with control mice. Liraglutide also improved liver morphology and reduced the accumulation of lipid droplets. Furthermore, the expression of glucose-6-phosphatase and phosphoenolpyruvate carboxykinase was downregulated, whereas the expression of phosphorylated forkhead box O1, Wnt signaling pathway-associated molecules, $\beta$-catenin, transcription factor 7-like 2 and phosphorylated glycogen synthase kinase-3 $\beta$ was upregulated in the liver of mice treated with liraglutide. In the in vitro study, increased gluconeogenesis and decreased glucose uptake rates were observed in insulin resistant hepatocytes; treatment with liraglutide significantly reversed this effect. Furthermore, transfection of insulin resistant hepatocytes with $\beta$-catenin small interfering RNA
\end{abstract}

Correspondence to: Dr Gang Yuan or Dr Dao-Wen Wang, Department of Internal Medicine, Tongji Hospital, Huazhong University of Science and Technology, 1095 Jiefang Road, Wuhan, Hubei 430030, P.R. China

E-mail: yuangang88@hotmail.com

E-mail: dwwang@tjh.tjmu.edu.cn

${ }^{*}$ Contributed equally

Key words: $\beta$-catenin, diabetes mellitus, glucagon-like peptide-1, gluconeogenesis, liver attenuated the effects of liraglutide, suggesting that liraglutide improves insulin resistance via activating the $\beta$-catenin/Wnt signaling pathway. The results of the present study suggest a novel mechanism underlying liraglutide-mediated improvements in insulin resistance in the liver. The Wnt signaling pathway may be a potential therapeutic target for the treatment of altered hepatic physiology in insulin resistance.

\section{Introduction}

Glucagon-like peptide-1 (GLP-1) is an incretin hormone encoded by the glucagon GCG gene (1). GLP-1 is secreted from the gastrointestinal tract into the circulation in response to nutrient ingestion $(1,2)$. GLP-1 stimulates insulin secretion from $\beta$-cells and inhibits glucagon secretion from $\alpha$-cells (3) and has previously been used for development of novel strategies to treat type 2 diabetes. In a clinical study involving patients with type 2 diabetes, administering a subcutaneous infusion of GLP-1 for 6 weeks resulted in improved insulin sensitivity and $\beta$-cell function (4). Ding et al (5) reported that GLP-1 decreased the levels of serum glucose and improved insulin resistance. Exendin-4, a GLP-1 receptor agonist, has been reported to inhibit the expression of glucose-6-phosphatase (G6Pase) and phosphoenolpyruvate carboxykinase (PEPCK) in the hepatocytes of young adult mice via an insulin-independent mechanism (6). Several studies have demonstrated that the GLP-1 receptor is a member of the 7 transmembrane family of $\mathrm{G}$ protein coupled receptors and is expressed in the human hepatocyte cell lines HepG2 and Huh7 as well as C57BL/6J mice with high-fat-diet-induced obesity (6-8). However, the exact mechanism underlying GLP-1-mediated improvement of liver insulin resistance remains to be elucidated.

Evolutionarily conserved proto-oncogene Wnt (Wnt) signaling pathways are present in multicellular eukaryotes and serve roles in embryonic development, morphogenesis, stem cell regulation, liver zonation and metabolism (9). Wnt genes encode 19 Wnt proteins and are evolutionarily conversed (9). To date, two different Wnt signaling pathways have been identified, including the canonical and non-canonical pathway (10). The canonical Wnt signaling pathway components include the frizzled family of cell surface receptors, T-cell factor (TCF) family transcription factors, co-receptors and the downstream 
degradation complex containing axin/glycogen synthase kinase (GSK)-3ß/adenomatosis polyposis coli (APC) and $\beta$-catenin (11-13). Interactions between Wnt and frizzled receptors and/or co-receptors, including low-density lipoprotein receptor-related protein (LRP) 5 and 6, leads to the activation of the intracellular protein disheveled (Dsh). These events trigger an intracellular signaling cascade, which eventually results in phosphorylation and inactivation of glycogen synthase kinase-3 $\beta$ (GSK3 $\beta$ ), and decreased GSK3 $\beta$-induced phosphorylation of $\beta$-catenin (14). Accumulation and nuclear translocation of $\beta$-catenin, and increased levels of nuclear $\beta$-catenin lead to the activation of over 60 Wnt-responsive genes via interactions with transcription factors, including TCF, TCF7, TCF7 like 1 (TCF7L1), TCF7L2 and lymphoid enhancer factor (LEF) (14).

Genetic associations between TCF7L2 polymorphisms and type 2 diabetes has previously been reported in multiple ethnic populations (15). Several studies have investigated the role of the Wnt signaling pathway in the pathogenesis of diabetes (16-19). In TCF7L2 gene knockout mice, the number of pancreatic $\beta$-cells was significantly reduced along with the reduction of the deficiencies in insulin secretion and glucose tolerance (16). Liver-specific TCF7L2 knockout mice demonstrated increased hepatic glucose production and overexpression of liver-specific TCF4, leading to reduced hepatic glucose levels (17). TCF7 knockdown in HepG2 cells resulted in increased gluconeogenic gene expression, whereas restoration of hepatic TCF7 levels decreased the expression of gluconeogenic genes and reduced hepatic glucose output (18). Using the liver-specific dominant-negative TCF7L2 transgenic mouse model LTCFDN, Ip et al (19) demonstrated that the Wnt signaling pathway and its effector $\beta$-catenin/TCF serve a beneficial role in suppressing hepatic gluconeogenesis.

GLP-1 has been demonstrated to directly activate the Wnt signaling pathway (20). Liu and Habener (21) reported that GLP-1 and exendin-4 induced Wnt signaling in pancreatic $\beta$-cells and INS-1 cells independent of GSK3 $\beta$, as well as increasing nuclear levels of $\beta$-catenin. These interactions led to transcriptional activation of genes associated with $\beta$-cell proliferation via interacting with TCF7L2 (21). Furthermore, it has been demonstrated that lithium, a GSK3 $\beta$ inhibitor, stimulates the synthesis of GLP-1 and activates the transcription of proglucagon in intestinal endocrine cell lines (14). These observations suggest that Wnt signaling effectors $\beta$-catenin and TCF are able to control the production and function of GLP-1 in islets and insulin-secreting INS-1 cells (21). However, it has not yet been investigated whether GLP-1 improves liver insulin resistance via the Wnt signaling pathway.

The aim of the present study was to investigate whether the Wnt signaling pathway mediates the beneficial effects of liraglutide on insulin resistance and hepatic glucose metabolism using in vitro and in vivo diabetic models. The results of the present study demonstrate that the interaction between forkhead box (Fox) O1, the main target of insulin signaling, and $\beta$-catenin regulates hepatic gluconeogenesis in response to liraglutide.

\section{Materials and methods}

Animal studies. Male db/db mice ( $\mathrm{n}=12,40-44 \mathrm{~g})$ and C57BL/6 mice ( $\mathrm{n}=6,39-42 \mathrm{~g}$ ) were purchased from the Institute for Animal Reproduction of Experimental Animal Center in
Nanjing Medical College (Nanjing, China). C57BL/6 mice were used as the control group. The mice were bred under standard laboratory conditions and used for experimentation when 6 weeks old. All animals were maintained in a temperature-controlled environment $\left(22-24^{\circ} \mathrm{C} ; 50-60 \%\right.$ humidity) with a 12-h light/dark cycle and free access to drinking water and normal chow $(60,15$ and $25 \%$ calories from carbohydrates, fats and proteins, respectively). All animal care and experimental procedures were approved by the Institutional Animal Care and Use Committee of Tongji Medical College, Huazhong University of Science and Technology (Wuhan, China). Male $\mathrm{db} / \mathrm{db}$ mice were divided into 2 groups: Saline and liraglutide ( $n=6 /$ group) and received intraperitoneal injections of saline or liraglutide (Novo Nordisk Pharmaceutical Co., Ltd., Tianjin, China) once daily at a concentration of $200 \mu \mathrm{g} / \mathrm{kg}$ body weight for 8 weeks. Animals were monitored each 2 weeks for alterations in body weight, plasma glucose and insulin. Every 2 weeks, the tail vein blood was harvested from the tail vein and plasma was stored at $-80^{\circ} \mathrm{C}$ for further analysis. A Glucose Oxidase and Peroxidase Assay (GOD-POD) kit (Shanghai Mingdian Bioengineering, Co., Ltd., Shanghai, China) and an Insulin ELISA kit (cat. no. EZHIASF-14K; EMD Millipore, Billerica, MA, USA) were used to quantify levels of glucose and insulin, respectively. The homeostasis model assessment for insulin resistance (HOMA-IR) was calculated using the following equation: Fasting insulinxfasting glucose/22.5 (22). Animals were fasted overnight in the 8th week of the experiment and subsequently sacrificed by cervical dislocation. Liver tissues were harvested, frozen in liquid nitrogen and stored at $-80^{\circ} \mathrm{C}$ until RNA and protein extraction.

Hematoxylin and eosin $(H \& E)$ staining. Liver tissues were fixed in $10 \%$ formalin for $24 \mathrm{~h}$ at room temperature and embedded in paraffin. Paraffin blocks were cut using a microtome into 5- $\mu \mathrm{m}$ sections. Following deparaffinization and dehydration, the sections were stained with $0.5 \%(\mathrm{w} / \mathrm{v})$ hematoxylin (5-10 min) and eosin (1-2 min; H\&E staining) at room temperature. Histological slides were examined by light microscopy (magnification, x200). Histological features, including steatosis, inflammation and hepatocellular injury were histologically graded by two pathologists. The nonalcoholic fatty liver disease (NAFLD) activity score (NAS) was quantified by summing the scores of steatosis (0-3), lobular inflammation (0-2) and hepatocellular ballooning (0-2), as previously described (23).

Cell culture. The HepG2 human hepatoma cell line was purchased from American Type Culture Collection (ATCC; Manassas, VA, USA). Cells were grown and cultured in Dulbecco's modified Eagle's medium (DMEM; Gibco; Thermo Fisher Scientific, Inc., Waltham, MA, USA) containing 10\% fetal bovine serum (FBS; Gibco; Thermo Fisher Scientific, Inc.), $10 \mathrm{mmol} / 1$ 4-(2-hydroxyethyl)-1-piperazineethanesulfonic acid (Sigma-Aldrich; Merck KGaA, Darmstadt, Germany), 100 U/ml penicillin, and $100 \mathrm{mg} / \mathrm{ml}$ streptomycin. Cells were maintained at $37^{\circ} \mathrm{C}$ in a humidified incubator, with $5 \% \mathrm{CO}_{2}$.

Insulin resistance cell model. At 70-80\% confluence, cells were treated with $250 \mu \mathrm{mol} / 1$ palmitate (Sigma-Aldrich; Merck $\mathrm{KGaA}$ ), one of the most common free fatty acids, 
and high glucose $(25 \mathrm{mmol} / \mathrm{l})$ DMEM culture medium for $24 \mathrm{~h}$ to establish the in vitro insulin resistant model. Glucose consumption studies were performed to evaluate the insulin resistant model. Cells were washed three times with PBS and the culture medium was replaced with low glucose DMEM ( $5.5 \mathrm{mmol} / \mathrm{lglucose})$. Insulin was added to the culture medium at a concentration of $10^{-7} \mathrm{~mol} / \mathrm{l}$ for $2 \mathrm{~h}$. Following incubation, culture medium was collected and tested for glucose consumption was investigated as previously described (24), using the GOD-POD kit according to the manufacturer's instructions.

Small interfering RNA (siRNA) transfection. Following successful establishment of the in vitro insulin resistant model, $\beta$-catenin siRNA (50 nM; Guangzhou RiboBio Co., Ltd., Guangzhou, China) was transfected into the cells via incubation with MegaTran 1.0 (OriGene Technologies, Inc., Rockville, MD, USA) for $48 \mathrm{~h}$ at $37^{\circ} \mathrm{C}$. $\beta$-catenin siRNA was diluted in opti-MEM (Gibco; Thermo Fisher Scientific, Inc.) to obtain a final concentration of $100 \mathrm{nmol} / \mathrm{l}$. At $24 \mathrm{~h}$ following transfection, $100 \mathrm{nM}$ liraglutide was added to cell cultures for $24 \mathrm{~h}$. HepG2 cells were harvested and used for western blot analysis. Following incubation, culture medium was collected and glucose consumption was investigated as previously described (24), using the GOD-POD kit according to the manufacturer's instructions.

Glucose production assay. HepG2 cells were plated in 24-well cell culture plates at a density of $2 \times 10^{5}$ cells/well and allowed to grow for $24 \mathrm{~h}$ at $37^{\circ} \mathrm{C}$. HepG2 cells were transfected with $\beta$-catenin siRNA as above. Subsequently, cells were washed three times with PBS to remove glucose and treated for $24 \mathrm{~h}$ with glucose production medium (glucose- and phenol red-free DMEM containing gluconeogenesis substrates: $10 \mathrm{mM}$ sodium lactate and $1 \mathrm{mM}$ sodium pyruvate), $0.1 \mathrm{mM}$ adenosine 3',5'-cyclic monophosphate (cAMP) and $100 \mathrm{nM}$ dexamethasone (Dex) to induce gluconeogenesis in the absence or presence of $100 \mathrm{nM}$ liraglutide. Glucose production was measured using a Glucose Assay kit (Shanghai Mingdian Bioengineering, Co., Ltd.) according to the manufacturer's instrucctions.

Western blot analysis. HepG2 cells were lysed in radioimmunoprecipitation assay buffer (RIPA; Beyotime Institute of Biotechnology, Beijing, China) containing 1\% Triton X-100 and a mixture of protease and phosphatase inhibitors. Liver tissue (30 mg wet weight) was homogenized at $4^{\circ} \mathrm{C}$ in $400 \mu 1$ RIPA buffer containing 1\% Triton X-100 and a mixture of protease and phosphatase inhibitors. Following a 30 min-incubation on ice, total protein was obtained by centrifugation at $4,025 \mathrm{x}$ g for $20 \mathrm{~min}$ at $4^{\circ} \mathrm{C}$. Protein content was measured using the bicinchoninic acid protein assay and $30 \mu \mathrm{g}$ protein was separated by $10 \%$ SDS-PAGE. Separated proteins were electrically transferred onto polyvinylidene difluoride membranes. Following transfer, membranes were blocked with $5 \%$ bovine serum albumin (MP Biomedicals, LLC, Santa Ana, CA, USA) for $1 \mathrm{~h}$ at room temperature, washed in Tris-buffered saline with $0.08 \%$ Tween and then incubated overnight at $4^{\circ} \mathrm{C}$ with the following primary antibodies: $\beta$-catenin (1:1,000; cat. no. sc-7963), TCF7L2 (1:1,000; cat. no. sc-166699), PEPCK (1:1.000; cat. no. sc-32879), G6Pase (1:1,000; cat. no. sc-25840), GSK3 $\beta$ (1:1,000; cat. no. sc-81462),
phospho-Ser473-Akt (1:1,000; cat. no. sc-33437), total Akt (1:1,000; cat. no. sc-8312), $\beta$-actin (1:2,000; cat. no. sc-47778; all from Santa Cruz Biotechnology, Inc., Dallas, TX, USA), p-Ser9-GSK3 $\beta$ (1:1,000; cat. no. 9323), p-Ser256-FoxO1 (1:1,000; cat. no. 9461) and total FoxO1 (1:1,000; cat. no. 2880; all from Cell Signaling Technology, Danvers, MA, USA). The membranes were washed, and incubated at room temperature for $1 \mathrm{~h}$ with the following secondary antibodies: Horseradish peroxidase (HRP)-conjugated sheep anti-rabbit immunoglobulin $(\operatorname{Ig}) \mathrm{G}$ (1:2,000; cat. no. SA00001-2; ProteinTech Group, Inc., Chicago, IL, USA) or HRP-conjugated goat anti-mouse IgG (1:2,000; cat. no. SA00001-1; ProteinTech Group, Inc.). Proteins were visualized using enhanced chemiluminescent detection reagent (Beyotime Institute of Biotechnology), and quantified using Quantity One software (version 4.6.2; Bio-Rad Laboratories, Inc., Hercules, CA, USA).

\section{Reverse transcription-quantitative polymerase chain reaction} $(R T-q P C R)$. Total RNA was extracted from liver tissue using TRIzol reagent (Invitrogen; Thermo Fisher Scientific, Inc.) according to the manufacturer's instructions. cDNA was synthesized using an RT-qPCR kit (cat. no. 10928042; Invitrogen; Thermo Fisher Scientific, Inc.). qPCR was performed to detect mouse $\beta$-catenin, TCF7L2, PEPCK, G6Pase, FoxO1 and GSK3 $\beta$ using QuantiTect SYBR Green PCR kit (Applied Biosystems; Thermo Fisher Scientific, Inc.) and a real-time PCR detection system (Bio-Rad Laboratories, Inc.). The following primer pairs were used for the qPCR: $\beta$-catenin forward, 5 -GTTCTACGC CATCACGACAC-3' and reverse, 5'-GACAGCACCTTCAGC ACTCT-3'; TCF7L2 forward, 5'-AATCCTTGCCTTTCGCTT CC-3' and reverse, 5'-TCTGTGACTTGGCGTCTTGG-3'; pck1 forward, 5'-ACAGTCATCATCACCCAAGAGC-3' and reverse, 5'-GGGCGAGTCTGTCAGTTCAATA-3'; G6Pase forward, 5'-CATCAATCTCCTCTGGGTGGC-3' and reverse, 5'-GCT GTTGCTGTAGTAGTCGGTGTC-3'; FoxO1 forward, 5'-TGC TTTTATTACCCTGTGAGTTGTG-3' and reverse, 5'-ATC GTGACAAAAGCCAACAGC-3'; Gsk3 $\beta$ forward, 5'-GGT CAGTTTCACAGGGTTATGC-3' and reverse, 5'-AGATGG AAGTGGTCACGCTAAT-3'; $\beta$-actin forward, 5'-AGAGGG AAATCGTGCGTGAC-3' and reverse, 5'-CAATAGTGATGA CCTGGCCGT-3'. The following thermocycling conditions were used for the PCR: Initial denaturation at $95^{\circ} \mathrm{C}$ for $5 \mathrm{~min}$ followed by 40 cycles of $95^{\circ} \mathrm{C}$ for $15 \mathrm{sec}, 60^{\circ} \mathrm{C}$ for $15 \mathrm{sec}$ and $72^{\circ} \mathrm{C}$ for $20 \mathrm{sec}$. Relative quantification was performed using the $2^{-\Delta \Delta C q}$ method (25). Transcript levels were normalized to the internal reference gene $\beta$-actin.

Statistical analysis. Data are presented as the mean \pm standard error of the mean. Statistical comparisons were performed using the unpaired two-tailed t-test or one-way analysis of variance followed by Newman-Keuls post hoc test for multiple group analyses. Analysis was performed using SPSS 17.0 software (SPSS, Inc., Chicago, IL, USA). P $<0.05$ was considered to indicate a statistically significant difference.

\section{Results}

Liraglutide significantly decreases body weight, plasma glucose concentration, insulin levels and HOMA-IR. The body weight of $\mathrm{db} / \mathrm{db}$ mice treated with liraglutide decreased 
A

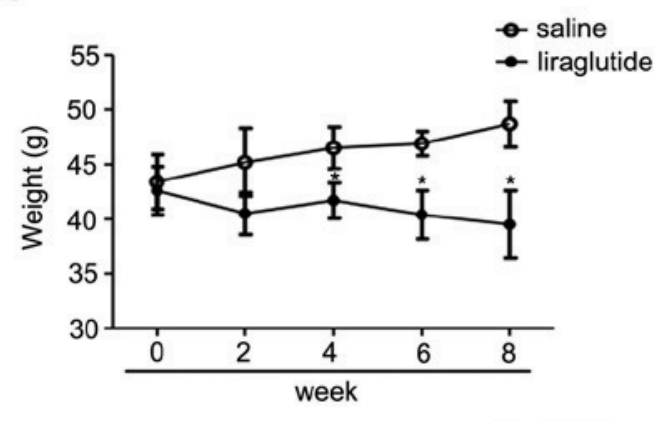

C

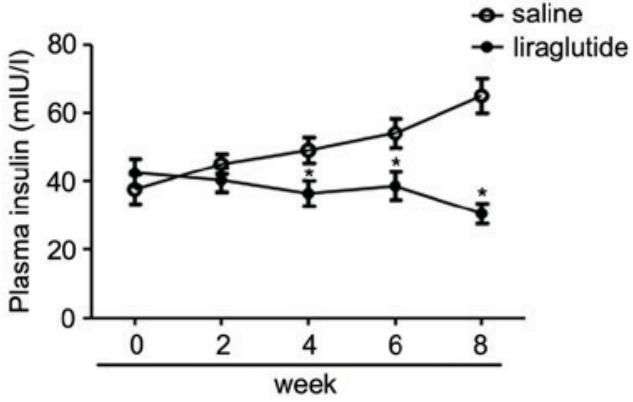

B
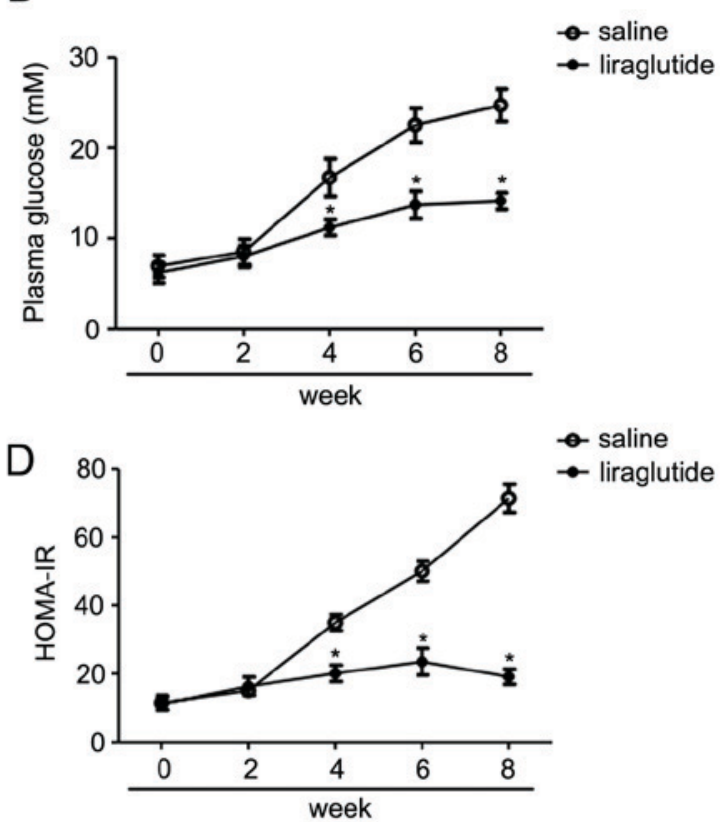

$\mathrm{E}$
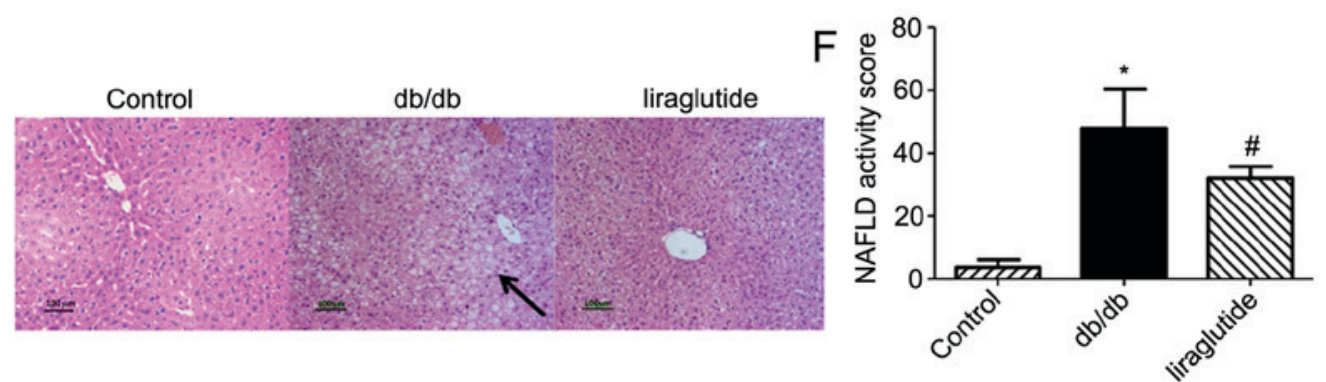

Figure 1. Liraglutide significantly decreases body weight, plasma glucose concentration, insulin and HOMA-IR and alters the liver morphology. (A) Mean body weight, (B) plasma glucose levels, (C) plasma insulin levels and (D) HOMA-IR following treatment with liraglutide or saline. (E) Hematoxylin and eosin staining of liver tissue. The arrow indicates lipid droplets (magnification, x200). (F) Histopathological analysis was conducted by quantifying NAFLD activity score. All values are presented as the mean \pm standard error of the mean. ${ }^{*} \mathrm{P}<0.05$ vs. control; ${ }^{*} \mathrm{P}<0.05 \mathrm{vs}$. db/db group. HOMA-IR, homeostasis model assessment for insulin resistance; NAFLD, non-alcoholic fatty liver disease.

from week 4 onwards compared with the saline-treated control group (all $\mathrm{P}<0.05$; Fig. 1A). The final body weight of the control group at week 8 was significantly increased compared with the liraglutide treated group $(39.5 \pm 3.1 \mathrm{~g}$ in the liraglutide treated group vs. $48.7 \pm 2.1 \mathrm{~g}$ in the control group; $\mathrm{P}<0.05$ ).

Quantification of plasma glucose levels revealed significantly reduced glucose in the liraglutide treated group $(14.1 \pm 0.9 \mathrm{mM})$, compared with the control group $(24.7 \pm 1.8 \mathrm{mM})$ at week $8(\mathrm{P}<0.05$; Fig. 1B). Similarly, at week 8 the plasma insulin concentration in the liraglutide treated group (30.5 $\pm 2.9 \mathrm{mIU} / \mathrm{l})$ was significantly lower compared with the saline treated group $(65.0 \pm 5.4 \mathrm{mIU} / \mathrm{l} ; \mathrm{P}<0.05$; Fig. 1C). Treatment with liraglutide significantly improved HOMA-IR insulin resistance $(19.1 \pm 2.2)$, compared with the control group (71.4 $\pm 4.1 ; \mathrm{P}<0.05$; Fig. 1D).

Liraglutide alters liver morphology. Hepatic accumulation of lipids increased in the $\mathrm{db} / \mathrm{db}$ group compared with control group and decreased in mice treated with liraglutide (Fig. 1E). Additionally, NAFLD activity significantly decreased in the liraglutide group compared with the saline group $(\mathrm{P}<0.05$; Fig. $1 \mathrm{~F})$.
Liraglutide activates the Wnt signaling pathway and downregulates liver gluconeogenesis in vivo. The present study aimed to identify the signaling pathway and potential mechanisms underlying the effects of liraglutide on insulin resistance. GLP-1 is an activator of the canonical Wnt signaling pathway; as such, changes in the expression of $\beta$-catenin, GSK $3 \beta$ and TCF7L2 in the liver samples were analyzed using western blotting and RT-qPCR. Levels of $\beta$-catenin protein, but not mRNA, increased significantly in the $\mathrm{db} / \mathrm{db}$ group treated with liraglutide compared with the control group treated with saline $(\mathrm{P}<0.05$; Fig. 2A-C). Furthermore, levels of TCF7L 2 mRNA and protein, levels of GSK $3 \beta$ mRNA, and levels of p-GSK3 $\beta$ protein were significantly increased in liraglutide-treated mice compared with the saline group $(\mathrm{P}<0.05$; Fig. 2A-C). Furthermore, treatment with liraglutide significantly inhibited kinase enzymes G6Pase and PEPCK at the mRNA and protein level (all $\mathrm{P}<0.05$; Fig. 2A-C). Upstream of PEPCK and G6Pase, FoxO1 is a transcription factor that promotes gluconeogenesis in the liver, whereas p-FoxO1 suppresses gluconeogenesis (26). The levels of total and p-FoxO1 were assessed in the present study (Fig. 2A-C). The results demonstrated that the levels of $\mathrm{p}-\mathrm{FoxO} 1$ protein 


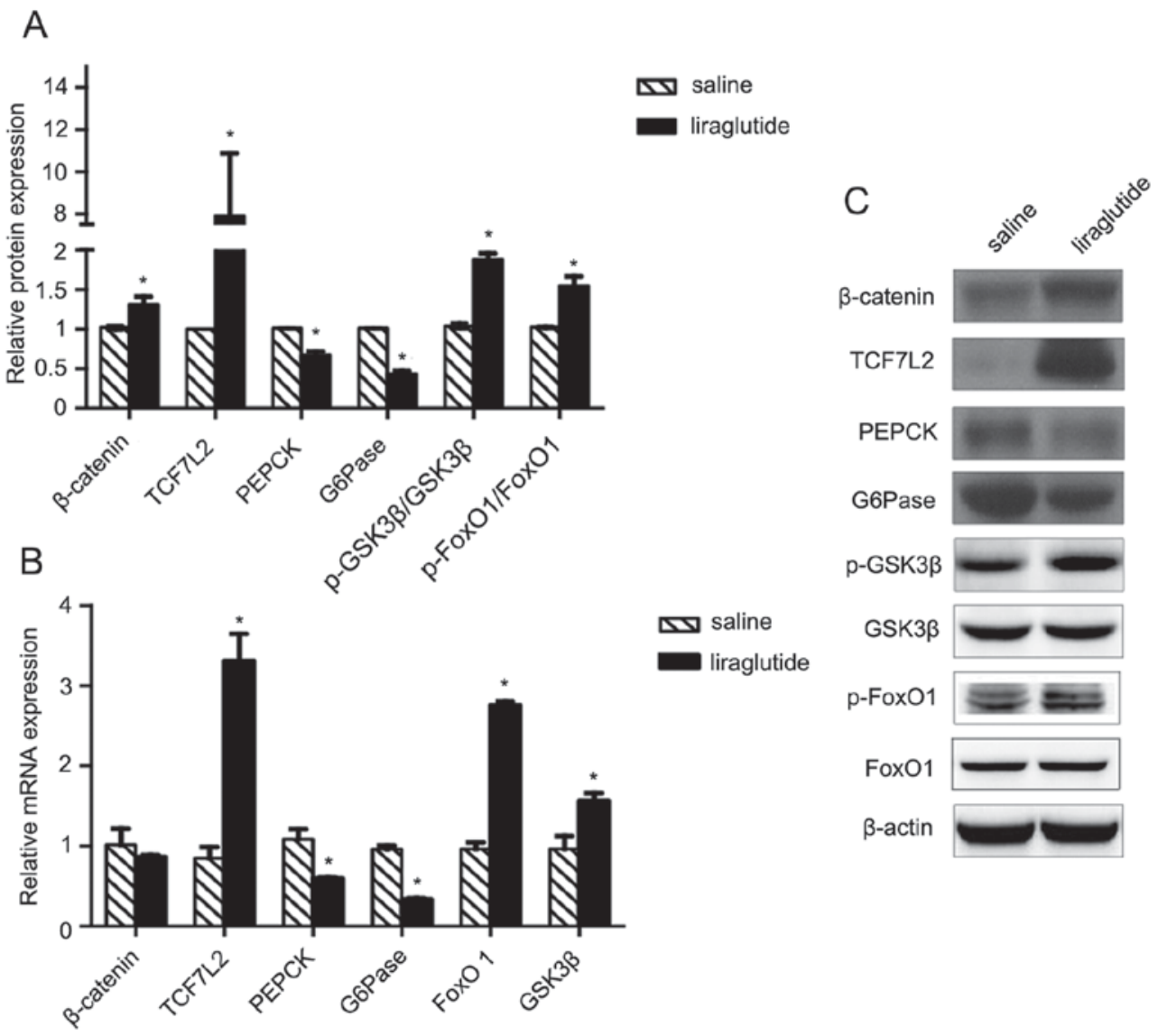

Figure 2. Liraglutide activates the Wnt signaling pathway and decreases liver gluconeogenesis in vivo. (A) Protein and (B) mRNA levels of Wnt signaling pathway molecules, including $\beta$-catenin, TCF7L2 and p-GSK3 $\beta$, and molecules associated with liver gluconeogenesis, including PEPCK, G6Pase and p-FoxO1. $\beta$-actin was used as a loading control. (C) Representative western blot images of the Wnt signaling pathway molecules and molecules associated with liver gluconeogenesis. All values are expressed as the mean \pm standard error of the mean. ${ }^{*} \mathrm{P}<0.05$ vs. control. TCF7L2, transcription factor 7 like 1 ; p, phosphorylated; GSK3 $\beta$, glycogen synthase kinase-3 $\beta$; PEPCK, phosphoenolpyruvate carboxykinase; G6Pase, glucose-6-phosphatase; FoxO1, forkhead box O1; Wnt, proto-oncogene Wnt.

and therefore the ratio of $\mathrm{p}$-FoxO1/FoxO1 were significantly elevated in liraglutide treated $\mathrm{db} / \mathrm{db}$ mice compared with saline-treated mice $(\mathrm{P}<0.05)$. These results suggest that liraglutide inactivates FoxO1 by phosphorylation and therefore inhibits the downstream activity of G6Pase and PEPCK. The above data indicate that liraglutide improves insulin resistance and reduces liver gluconeogenesis; it may be hypothesized that these effects are mediated by the $\beta$-catenin/Wnt signaling pathway.

Liraglutide increases glucose uptake and decreases glucose production in hepatocytes, but this effect is reversed in hepatocytes transfected with $\beta$-catenin siRNA. To investigate the effect of $\beta$-catenin on liraglutide-induced decrease in insulin resistance in vitro, $\mathrm{HepG} 2$ human hepatocytes were transfected with $\beta$-catenin siRNA for $48 \mathrm{~h}$. The transfection efficiency was subsequently determined by verifying $\beta$-catenin protein levels using western blot analysis (Fig. 3A). The results demonstrate that the expression of $\beta$-catenin was significantly reduced in transfected cells $(1.27 \pm 0.15)$ compared with the control cells $(7.44 \pm 0.40 ; \mathrm{P}<0.05)$.

The effects of liraglutide on glucose uptake were investigated by establishing an in vitro insulin resistance model in palmitate- and high glucose-stimulated human hepatocytes.
The amount of glucose in cell culture supernatants was quantified by the GOD-POD assay. Insulin resistant cells had a lower glucose uptake rate compared with the control cells $(\mathrm{P}<0.05$, Fig. $3 \mathrm{~A})$. Following treatment with liraglutide, the rate of glucose uptake increased significantly compared with the insulin resistant cells $(\mathrm{P}<0.05)$. Furthermore, silencing $\beta$-catenin resulted in a decreased glucose uptake rate $(\mathrm{P}<0.05)$; however, treatment with liraglutide in $\beta$-catenin silenced cells had no effect on glucose uptake (Fig. 3B). In the glucose production experiment, liraglutide significantly suppressed cAMP+Dex-induced glucose production $(\mathrm{P}<0.05$; Fig. $3 C)$. Silencing $\beta$-catenin resulted in increased glucose production in the liraglutide treated group compared with the control $(\mathrm{P}<0.05)$. These results indicate that liraglutide increased glucose uptake and suppressed glucose production in hepatocytes via a $\beta$-catenin dependent mechanism.

Liraglutide activates the Wnt signaling pathway and decreases liver gluconeogenesis in vitro. To investigate whether the effects of liraglutide are mediated by the Wnt signaling pathway in vitro, HepG2 cells were transfected with $\beta$-catenin siRNA and treated with or without $100 \mathrm{nM}$ liraglutide. Western blot analysis revealed that liraglutide increased the expression of $\beta$-catenin protein and that this effect was reversed following 
A

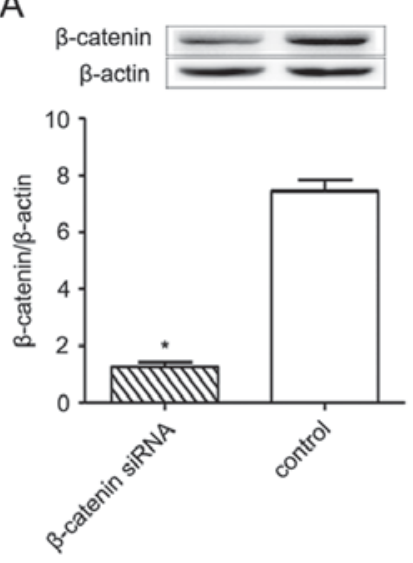

B

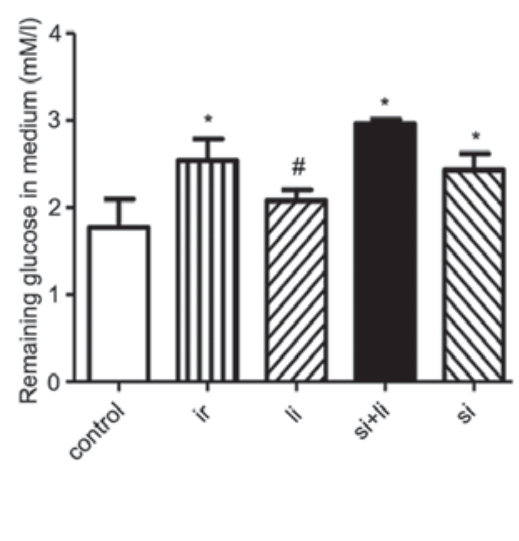

C

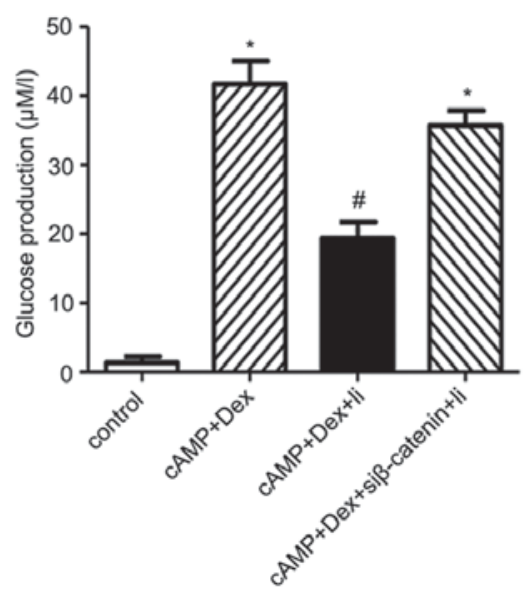

Figure 3. Liraglutide increases glucose uptake and decreases glucose production in hepatocytes; this effect is reversed in $\beta$-catenin siRNA transfected hepatocytes. (A) Transfection efficiency was detected by assessing $\beta$-catenin protein levels using western blot analysis. Blots are representative of three independent experiments with similar results. (B) Glucose uptake assay and (C) glucose production assay. All values are expressed as the mean \pm standard error of the mean. ${ }^{*} \mathrm{P}<0.05$ vs. control and ${ }^{\#} \mathrm{P}<0.05$ vs. the ir group. Ir, insulin resistance; li, liraglutide; si, $\beta$-catenin small interfering RNA; cAMP, cyclic adenosine 3',5'-monophosphate; dex, dexamethasone.

A

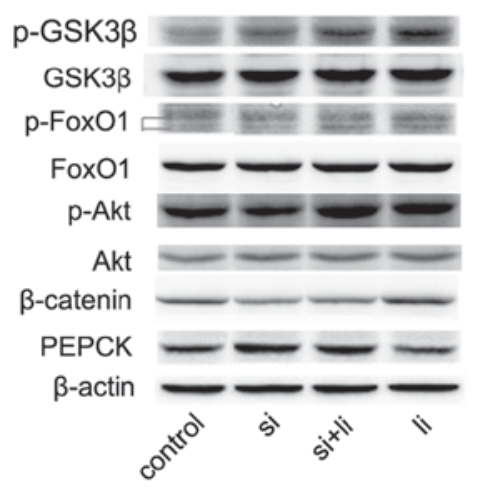

D

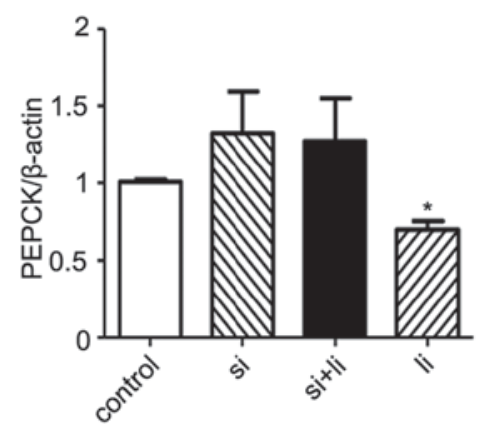

B

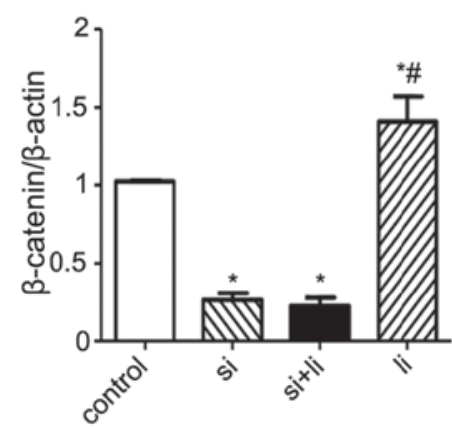

$\mathrm{E}$

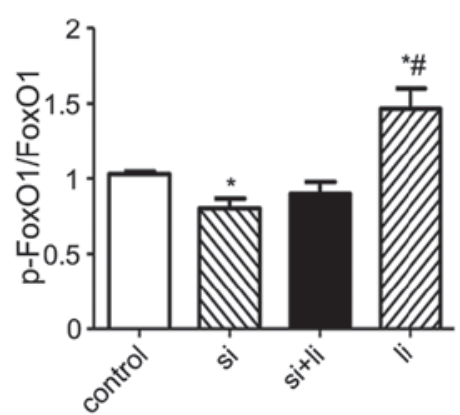

C

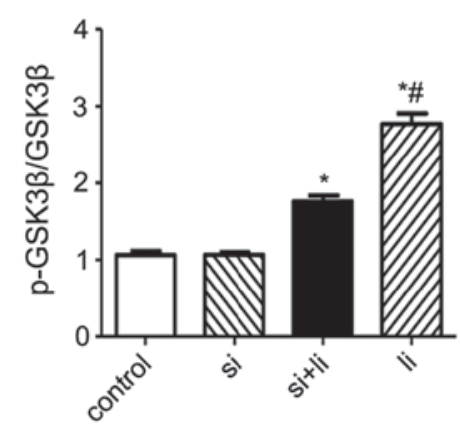

$\mathrm{F}$

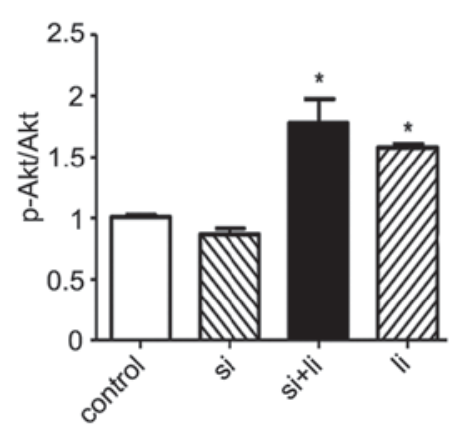

Figure 4. Liraglutide activates the Wnt signaling pathway and decreases liver gluconeogenesis in vitro. (A) Representative western blot images of the Wnt signaling pathway molecules, insulin signaling pathway molecules and molecules associated with liver gluconeogenesis. Protein levels of Wnt signaling pathway molecules, including (B) $\beta$-catenin and (C) total/p-GSK3 $\beta$. Expression levels of liver gluconeogenesis molecules, including (D) PEPCK and (E) total/pFoxO1. Expression levels of insulin signaling pathway molecules, including (F) p-AKT/Akt. The blots are representative of three independent experiments with similar results. $\beta$-actin was used as a loading control. All values are expressed as the mean \pm standard error of the mean. ${ }^{*} \mathrm{P}<0.05$ vs. con and ${ }^{\sharp} \mathrm{P}<0.05$ vs. si. Ir, insulin resistance; li, liraglutide; si, $\beta$-catenin small interfering RNA; Wnt, proto-oncogene Wnt; TCF7L2, transcription factor 7 like 1; p, phosphorylated; GSK3 $\beta$, glycogen synthase kinase-3 $\beta$; PEPCK, phosphoenolpyruvate carboxykinase; G6Pase, glucose-6-phosphatase; FoxO1, forkhead box O1; Akt, RAC-alpha serine/threonine-protein kinase.

$\beta$-catenin silencing $(\mathrm{P}<0.05$; Fig. $4 \mathrm{~A}$ and $\mathrm{B})$. Furthermore, as demonstrated in Fig. 4A and C, liraglutide significantly increased the $\mathrm{p}-\mathrm{GSK} 3 \beta / \mathrm{GSK} 3 \beta$ ratio, $(\mathrm{P}<0.05)$. The effect of liraglutide on GSK3 $\beta$ was partially inhibited in cells 
transfected with $\beta$-catenin siRNA. Treatment with liraglutide resulted in significantly decreased PEPCK protein expression compared with the control group; however, PEPCK expression was increased following transfection with $\beta$-catenin siRNA (both $\mathrm{P}<0.05$; Fig. 4A and D). Furthermore, the p-FoxO1/FoxO1 ratio was significantly increased in liraglutide-treated hepatocytes compared with both control and $\beta$-catenin siRNA transfected cells (both $\mathrm{P}<0.05$; Fig. $4 \mathrm{~A}$ and $\mathrm{E}$ ). Treatment with liraglutide in $\beta$-catenin-silenced cells decreased the ratio of $\mathrm{pFoxO} / \mathrm{FoxO}$, suggesting that liraglutide exerted its effects on FoxO1 and PEPCK via the canonical Wnt signaling pathway. Additionally, the levels of pAKT and total AKT, which are associated with the insulin signaling pathway, were analyzed (Fig. 4A and F). Western blot analysis indicated that liraglutide significantly increased the pAKT/AKT ratio compared with the control group $(\mathrm{P}<0.05)$. Transfection of hepatocytes with $\beta$-catenin siRNA had no significant effect on the ratio of pAKT/AKT, which remained similar to that identified in liraglutide-only treated cells. These results suggest that liraglutide acts mainly via the canonical $\beta$-catenin Wnt signaling pathway in hepatocytes.

\section{Discussion}

Liraglutide, a GLP-1 receptor agonist, is used for the treatment of diabetes. In addition to stimulating insulin secretion, liraglutide confers cardio-protective effects, suppresses appetite and delays gastric emptying (27,28). In patients with type 2 diabetes, liraglutide decreases the concentration of plasma glucose, hemoglobin A1c, fructosamine and free fatty acids, as well as improving insulin sensitivity and $\beta$-cell function (4). Furthermore, liraglutide inhibits glucagon release, improves insulin resistance and protects other organs, including the brain, cardiac tissue and muscles, from high glucose in an insulin-independent manner $(3,29,30)$. The results of the present study demonstrate that liraglutide markedly decreases plasma glucose, insulin, body weight and HOMA-IR. HOMA-IR is a method used to assess insulin resistance, which is inversely associated with insulin sensitivity as measured using an euglycemic clamp (22). Su et al (31) demonstrated that GLP-1 significantly decreased HOMA-IR in $\mathrm{db} / \mathrm{db}$ mice. Lee et al (8) reported that exendin-4 improves lobular inflammation and markedly reduces the accumulation of lipids, including free fatty acids and triglycerides in the fatty liver of high fat diet-induced obese C57BL/6J mice, thereby improving hepatic steatosis. In the present study, H\&E staining of liver specimens from $\mathrm{db} / \mathrm{db}$ mice treated with liraglutide revealed improved lobular structure, with reduced lobular inflammation and fewer lipid droplets within cells compared with the saline treated control group.

The liver regulates glucose metabolism and expresses the GLP-1 receptor (6). Numerous studies have demonstrated that GLP-1 reduces glucose production, gluconeogenesis, fatty acid synthesis and oxidative stress, as well as improving insulin resistance $(5,32)$. However, the mechanism underlying GLP-1-mediated improvements in insulin resistance remains to be elucidated. The therapeutic effect of GLP-1 against insulin resistance may be secondary to the effects of weight loss and attenuation of hyperglycemia (32). Previous studies have also demonstrated that GLP-1 may affect insulin resistance directly. Lee et al (8) reported that GLP-1 improved liver insulin resistance via activation of the sirtuin 1/adenosine monophosphate-activated protein kinase signaling pathway in C57BL/6J mice and HepG2 and Huh7 cell lines, whilst also increasing the expression of phosphorylated FoxO1 and GLUT2. GLP-1 promotes glycogen synthesis via activation of the phosphatidylinositol 4,5-bisphosphate 3-kinase/AKT signaling pathway in primary rat hepatocytes and this effect of GLP-1 is similar to that induced by insulin in liver (33). In HepG2 cells, silencing FoxO1 resulted in reduced expression of PEPCK and G6Pase (34). GLP-1 inhibited the expression of G6Pase and PEPCK in the liver and increased the phosphorylation of AKT and FoxO1 in young mice (6). In the present study, treatment with liraglutide resulted in decreased expression of PEPCK and G6Pase and increased p-FoxO1 levels compared with the control group. PEPCK and G6Pase serve roles in liver gluconeogenesis and decreased levels of these enzymes reduce the production of glucose, suggesting that liraglutide may inhibit the gluconeogenesis pathway. FoxO1 activates the expression of both PEPCK and G6Pase, whereas p-FoxO1 is targeted for proteasomal degradation (26). Furthermore, liraglutide-induced $\mathrm{p}$-FoxO1 may also contribute to the suppression of gluconeogenesis. These observations support the results obtained in a previous study, which reported that GLP-1 improved insulin resistance via inhibiting PEPCK and G6Pase (6).

The Wnt signaling pathway in the liver is associated with diabetes, glucose and lipid metabolism $(35,36)$. GLP-1 binds the GLP-1 receptor on cell membranes and activates cAMP-dependent protein kinase A (PKA) via the secondary messenger cAMP. GLP-1-mediated activation of PKA transmits the signal intracellularly and phosphorylates $\beta$-catenin to activate the canonical Wnt pathway (14). Activation of the Wnt signaling pathway induces the transcription of genes, including a diabetes risk gene TCF7L2 that stimulates the proliferation of pancreatic $\beta$ cells $(14,20,21,37)$. TCF7L2 silencing results in significantly increased expression of PEPCK and G6Pase $(35,38)$. FoxO1 and TCF7L 2 competitively bind $\beta$-catenin to regulate the hepatic metabolism of glucose. Under starvation, FoxO1 competes with TCF7L2 and binds $\beta$-catenin, which leads to increased gluconeogenesis (19). Insulin inhibits FoxO1 by stimulating its phosphorylation, thereby allowing $\beta$-catenin to bind TCF7L2 and inhibit liver gluconeogenesis $(39,40)$. In the present study, markedly increased levels of Wnt signaling pathway molecules TCF7L2, $\beta$-catenin and phosphorylated GSK3 $\beta$ were identified in mice treated with liraglutide, indicating a potential association with liver glucose metabolism under diabetic conditions. Db/db mice demonstrate $>10$-fold levels of plasma leptin compared with wild-type mice, which may also contribute to insulin resistance. GLP-1 markedly decreases levels of plasma leptin in $\mathrm{db} / \mathrm{db}$ mice $(31,41)$. It is therefore possible that the effect of liraglutide on liver gluconeogenesis in the present study may result from decreased leptin levels. To study the association between liraglutide and the Wnt signaling pathway, an in vitro insulin resistance cell model transfected with $\beta$-catenin siRNA was also used in the present study. The results demonstrate that treatment with liraglutide increases p-FoxO1 expression whilst downregulating PEPCK and the glucose uptake rate; $\beta$-catenin silencing effectively reversed these effects. The results of 
the present study suggest that liraglutide improves insulin resistance by increasing glucose uptake via $\beta$-catenin/Wnt signaling.

Levels of pAKT/AKT, which serve a role in the insulin signaling pathway, were investigated. The results of the present study demonstrate that liraglutide markedly increases the ratio of pAKT/AKT compared with control hepatocytes. Silencing $\beta$-catenin had no effect on the $\mathrm{pAKT} / \mathrm{AKT}$ ratio, suggesting that the effect of liraglutide on AKT phosphorylation is not mediated by $\beta$-catenin/Wnt signaling. AKT acts upstream of GSK $3 \beta$ and $\beta$-catenin. Therefore, liraglutide may participate in the insulin pathway by activating the canonical Wnt signaling pathway via increased phosphorylation of GSK3 $\beta$ and not AKT $(42,43)$.

In conclusion, the present study demonstrated that liraglutide decreases plasma glucose levels, body weight and HOMA-IR in mice with diabetes. Liraglutide reduced lobular inflammation and accumulation of lipid droplets, increased $\beta$-catenin, TCF7L2 and pGSK3 $\beta$ expression levels and led to the inactivation of FoxO1 and its downstream target genes, PEPCK and G6Pase. In HepG2 cells, liraglutide increased glucose uptake and reduced hepatic gluconeogenesis; these effects were reversed in cells transfected with $\beta$-catenin siRNA. Therefore, the present study suggests that treatment with liraglutide may increase the rate of glucose uptake and improve liver insulin resistance by activating the canonical Wnt signaling pathway. The results of the present study provide a novel mechanism by which liraglutide may improve insulin resistance in the liver. Furthermore, targeting the canonical Wnt signaling may have therapeutic potential for the treatment of altered hepatic physiology in insulin resistance and type 2 diabetes.

\section{Acknowledgements}

The present study was supported by a grant from the National Natural Science Foundation of China (grant no. 81370941, YG.).

\section{References}

1. Baggio LL and Drucker DJ: Biology of incretins: GLP-1 and GIP. Gastroenterology 132: 2131-2157, 2007.

2. Chiang YT, Ip W and Jin T: The role of the Wnt signaling pathway in incretin hormone production and function. Front Physiol 3: 273, 2012

3. Salehi M, Aulinger B, Prigeon RL and D'Alessio DA: Effect of endogenous GLP-1 on insulin secretion in type 2 diabetes. Diabetes 59: 1330-1337, 2010.

4. Zander M, Madsbad S, Madsen JL and Holst JJ: Effect of 6-week course of glucagon-like peptide 1 on glycaemic control, insulin sensitivity, and beta-cell function in type 2 diabetes: A parallel-group study. Lancet 359: 824-830, 2002.

5. Ding X, Saxena NK, Lin S, Gupta NA and Anania FA: Exendin-4, a glucagon-like protein-1 (GLP-1) receptor agonist, reverses hepatic steatosis in ob/ob mice. Hepatology 43: 173-181, 2006.

6. Fan R, Kang Z, He L, Chan J and Xu G: Exendin-4 improves blood glucose control in both young and aging normal non-diabetic mice, possible contribution of beta cell independent effects. PLoS One 6: e20443, 2011.

7. Gupta NA, Mells J, Dunham RM, Grakoui A, Handy J, Saxena NK and Anania FA: Glucagon-like peptide-1 receptor is present on human hepatocytes and has a direct role in decreasing hepatic steatosis in vitro by modulating elements of the insulin signaling pathway. Hepatology 51: 1584-1592, 2010.

8. Lee J, Hong SW, Chae SW, Kim DH, Choi JH, Bae JC, Park SE, Rhee EJ, Park CY, Oh KW, et al: Exendin-4 improves steatohepatitis by increasing Sirtl expression in high-fat diet-induced obese C57BL/6J mice. PLoS One 7: e31394, 2012.
9. Thompson MD and Monga SP: WNT/beta-catenin signaling in liver health and disease. Hepatology 45: 1298-1305, 2007.

10. Ackers I and Malgor R: Interrelationship of canonical and non-canonical Wnt signalling pathways in chronic metabolic diseases. Diab Vasc Dis Res, Nov 1, 2017 (Epub ahead of print).

11. Shao W, Wang D, Chiang YT, Ip W, Zhu L, Xu F, Columbus J, Belsham DD, Irwin DM, Zhang $\mathrm{H}$, et al: The Wnt signaling pathway effector TCF7L2 controls gut and brain proglucagon gene expression and glucose homeostasis. Diabetes 62: 789-800, 2013.

12. Jin T and Liu L: The Wnt signaling pathway effector TCF7L2 and type 2 diabetes mellitus. Mol Endocrinol 22: 2383-2392, 2008.

13. Akiyama T: Wnt/beta-catenin signaling. Cytokine Growth Factor Rev 11: 273-282, 2000.

14. Xiong X, Shao W and Jin T: New insight into the mechanisms underlying the function of the incretin hormone glucagon-like peptide- 1 in pancreatic $\beta$-cells: The involvement of the Wnt signaling pathway effector $\beta$-catenin. Islets 4: 359-365, 2012.

15. Grant SF, Thorleifsson G, Reynisdottir I, Benediktsson R, Manolescu A, Sainz J, Helgason A, Stefansson H, Emilsson V, Helgadottir A, et al: Variant of transcription factor 7-like 2 (TCF7L2) gene confers risk of type 2 diabetes. Nat Genet 38: 320-323, 2006.

16. da Silva Xavier G, Mondragon A, Sun G, Chen L, McGinty JA, French PM and Rutter GA: Abnormal glucose tolerance and insulin secretion in pancreas-specific Tcf712-null mice. Diabetologia 55: 2667-2676, 2012.

17. Boj SF, van Es JH, Huch M, Li VS, José A, Hatzis P, Mokry M, Haegebarth A, van den Born M, Chambon P, et al: Diabetes risk gene and Wnt effector Tcf712/TCF4 controls hepatic response to perinatal and adult metabolic demand. Cell 151: 1595-1607,2012.

18. Kaur K, Vig S, Srivastava R, Mishra A, Singh VP, Srivastava AK and Datta M: Elevated hepatic miR-22-3p expression impairs gluconeogenesis by silencing the Wnt-responsive transcription factor Tcf7. Diabetes 64: 3659-3669, 2015.

19. Ip W, Shao W, Song Z, Chen Z, Wheeler MB and Jin T: Liver-specific expression of dominant-negative transcription factor 7-like 2 causes progressive impairment in glucose homeostasis. Diabetes 64: 1923-1932, 2015.

20. Gustafson B and Smith U: WNT signalling is both an inducer and effector of glucagon-like peptide-1. Diabetologia 51: 1768-1770, 2008.

21. Liu Z and Habener JF: Glucagon-like peptide-1 activation of TCF7L2-dependent Wnt signaling enhances pancreatic beta cell proliferation. J Biol Chem 283: 8723-8735, 2008.

22. Matthews DR, Hosker JP, Rudenski AS, Naylor BA, Treacher DF and Turner RC: Homeostasis model assessment: Insulin resistance and beta-cell function from fasting plasma glucose and insulin concentrations in man. Diabetologia 28: 412-419, 1985.

23. Zhang Z, Li B, Meng X, Yao S, Jin L, Yang J, Wang J, Zhang H, Zhang Z, Cai D, et al: Berberine prevents progression from hepatic steatosis to steatohepatitis and fibrosis by reducing endoplasmic reticulum stress. Sci Rep 6: 20848, 2016.

24. Yin J, Hu R, Chen M, Tang J, Li F, Yang Y and Chen J: Effects of berberine on glucose metabolism in vitro. Metabolism 51: 1439-1443, 2002.

25. Livak KJ and Schmittgen TD: Analysis of relative gene expression data using real-time quantitative PCR and the 2(-Delta Delta C(T)) method. Methods 25: 402-408, 2001.

26. Tikhanovich I, Cox J and Weinman SA: Forkhead box class $\mathrm{O}$ transcription factors in liver function and disease. J Gastroenterol Hepatol 28 (Suppl 1): S125-S131, 2013.

27. Desouza CV, Gupta N and Patel A: Cardiometabolic effects of a new class of antidiabetic agents. Clin Ther 37: 1178-1194, 2015.

28. van Can J, Sloth B, Jensen CB, Flint A, Blaak EE and Saris WH: Effects of the once-daily GLP-1 analog liraglutide on gastric emptying, glycemic parameters, appetite and energy metabolism in obese, non-diabetic adults. Int J Obes (Lond) 38: 784-793, 2014.

29. Nyström T, Gutniak MK, Zhang Q, Zhang F, Holst JJ, Ahrén B and Sjöholm A: Effects of glucagon-like peptide-1 on endothelial function in type 2 diabetes patients with stable coronary artery disease. Am J Physiol Endocrinol Metab 287: E1209-E1215, 2004.

30. Alcántara AI, Morales M, Delgado E, López-Delgado MI, Clemente F, Luque MA, Malaisse WJ, Valverde I and Villanueva-Peñacarrillo ML: Exendin-4 agonist and exendin(9-39)amide antagonist of the GLP-1(7-36)amide effects in liver and muscle. Arch Biochem Biophys 341: 1-7, 1997. 
31. Su H, He M, Li H, Liu Q, Wang J, Wang Y, Gao W, Zhou L, Liao J, Young AA and Wang MW: Boc5, a non-peptidic glucagon-like Peptide-1 receptor agonist, invokes sustained glycemic control and weight loss in diabetic mice. PLoS One 3: e2892, 2008.

32. Cho YM, Fujita Y and Kieffer TJ: Glucagon-like peptide-1: Glucose homeostasis and beyond. Annu Rev Physiol 76: 535-559, 2014.

33. Redondo A, Trigo MV, Acitores A, Valverde I and Villanueva-Peñacarrillo ML: Cell signalling of the GLP-1 action in rat liver. Mol Cell Endocrinol 204: 43-50, 2003.

34. Nerurkar PV, Nishioka A, Eck PO, Johns LM, Volper E and Nerurkar VR: Regulation of glucose metabolism via hepatic forkhead transcription factor 1 (FoxO1) by Morinda citrifolia (noni) in high-fat diet-induced obese mice. Br J Nutr 108: 218-228, 2012.

35. Ip W, Shao W, Chiang YT and Jin T: The Wnt signaling pathway effector TCF7L2 is upregulated by insulin and represses hepatic gluconeogenesis. Am J Physiol Endocrinol Metab 303: E1166-E1176, 2012.

36. Liu H, Fergusson MM, Wu JJ, Rovira II, Liu J, Gavrilova O, Lu T, Bao J, Han D, Sack MN and Finkel T: Wnt signaling regulates hepatic metabolism. Sci Signal 4: ra6, 2011.

37. Hino S, Tanji C, Nakayama KI and Kikuchi A: Phosphorylation of beta-catenin by cyclic AMP-dependent protein kinase stabilizes beta-catenin through inhibition of its ubiquitination. Mol Cell Biol 25: 9063-9072, 2005.
38. Norton L, Fourcaudot M, Abdul-Ghani MA, Winnier D, Mehta FF, Jenkinson CP and Defronzo RA: Chromatin occupancy of transcription factor 7-like 2 (TCF7L2) and its role in hepatic glucose metabolism. Diabetologia 54: 3132-3142, 2011.

39. Hoogeboom D, Essers MA, Polderman PE, Voets E, Smits LM and Burgering BM: Interaction of FOXO with beta-catenin inhibits beta-catenin/T cell factor activity. J Biol Chem 283: 9224-9230, 2008.

40. Arai T, Kano F and Murata M: Translocation of forkhead box $\mathrm{O} 1$ to the nuclear periphery induces histone modifications that regulate transcriptional repression of PCK1 in HepG2 cells. Genes Cells 20: 340-357, 2015.

41. Patel V, Joharapurkar A, Gandhi T, Patel K, Dhanesha N, Kshirsagar S, Dhote V, Detroja J, Bahekar R and Jain M: Omeprazole improves the anti-obesity and antidiabetic effects of exendin-4 in db/db mice (-4 db/db)*. J Diabetes 5: 163-171. 2013.

42. Yi F, Sun J, Lim GE, Fantus IG, Brubaker PL and Jin T: Cross talk between the insulin and Wnt signaling pathways: Evidence from intestinal endocrine L cells. Endocrinology 149: 2341-2351, 2008.

43. Sun J, Wang D and Jin T: Insulin alters the expression of components of the Wnt signaling pathway including TCF-4 in the intestinal cells. Biochim Biophys Acta 1800: 344-351, 2010. 